\title{
A new technique for multi-cell joint channel estimation in time division code division multiple access based on reduced rank singular value decomposition
}

\begin{abstract}
A new technique for multi-cell joint channel estimation (MCJCE) in time division code division multiple access based on singular value decomposition (SVD) reduced rank technique is proposed in this paper. MCJCE is one of the effective solutions to improve the mobile system performance throughout mitigate the inter-cell interference form the neighboring cells. The increasing complexity of multi-cell system model due to the additional processing of the interferer users will be solved by using SVD reduced rank technique, where a limited number of parameter that really need it to describe the channel matrix will be estimated. Two models of multi-cell approaches are discussed, the first one depended on reconstruct the convolutional midamble matrix of inactive users in serving cell by the strongest interferer users from the neighboring cells. The second one will be more inclusive to user traffic scenarios in mobile systems and will be expanding to contain all detected users. The simulation results prove the validity of the proposed reduced rank technique for precision channel estimation $(6.4$ and $5 \mathrm{~dB})$ and $(9$ and $7 \mathrm{~dB})$ for case 1 and 2 respectively; BER performance improvements over the conventional estimators.
\end{abstract}

Keyword: TD-SCDMA; Joint channel estimation (JCE); Multi-user joint detection (MUJD); Reduced rank techniques; SVD 